\title{
VARIUS-NTV: A Microarchitectural Model to Capture the Increased Sensitivity of Manycores to Process Variations at Near-Threshold Voltages $\ddagger$
}

\author{
Ulya R. Karpuzcu*, Krishna B. Kolluru ${ }^{\dagger}$, Nam Sung Kim ${ }^{\dagger}$, and Josep Torrellas* \\ ${ }^{*}$ University of Illinois Urbana-Champaign ${ }^{\dagger}$ University of Wisconsin Madison \\ \{rkarpu2,torrella\}@illinois.edu \\ $\{k k o l l u r u, n s k i m 3\} @ w i s c . e d u$
}

\begin{abstract}
Near-Threshold Computing (NTC), where the supply voltage is only slightly higher than the threshold voltage of transistors, is a promising approach to attain energy-efficient computing. Unfortunately, compared to the conventional SuperThreshold Computing (STC), NTC is more sensitive to process variations, which results in higher power consumption and lower frequencies than would otherwise be possible, and potentially a non-negligible fault rate.

To help address variations at NTC at the architecture level, this paper presents the first microarchitectural model of process variations for NTC. The model, called VARIUS-NTV, extends the existing VARIUS variation model. Its key aspects include: (i) adopting a gate-delay model and an SRAM cell type that are tailored to NTC, (ii) modeling SRAM failure modes emerging at NTC, and (iii) accounting for the impact of leakage in SRAM models. We evaluate a simulated $11 \mathrm{~nm}, 288$-core tiled manycore at both NTC and STC. The results show higher frequency and power variations within the NTC chip. For example, the maximum difference in on-chip tile frequency is $\approx 2.3 x$ at STC and $\approx 3.7 x$ at NTC. We also validate our model against an experimental chip.
\end{abstract}

Keywords-Process variations, Near-threshold voltage, Manycore architectures, SRAM fault models, Power constraints.

\section{INTRODUCTION}

Power or energy consumption is typically the primary concern in today's computer platforms, ranging from datacenters to handhelds. The main reason for their importance is that CMOS technology has long ago stopped scaling close to perfectly and, as a result, power density increases significantly with each technology generation. If we are to continue delivering scalable computing performance, we need to find new ways to compute more energy- and powerefficiently.

One way to attain higher energy efficiency is to reduce the supply voltage $\left(V_{d d}\right)$ to a value only slightly higher than a transistor's threshold voltage $\left(V_{t h}\right)$. This environment is called Near-Threshold Computing (NTC) [7], [13], [28] - as opposed to conventional Super-Threshold Computing (STC). $V_{d d}$ is a most powerful knob because it impacts both dynamic and static energy super-linearly. Current indications

\footnotetext{
$\ddagger$ This work was supported in part by the National Science Foundation under grant CCF-1012759 and CAREER Award CCF-0953603; DARPA under UHPC Contract Number HR0011-10-3-0007; DOE ASCR under Award Number DE-FC02-10ER2599; an IBM Faculty Award; and a generous gift from AMD.
}

suggest that NTC can decrease the energy per operation by several times over STC [7], [13]. A drawback is that it imposes a frequency reduction, which may be tolerable through more parallelism in the application. For parallel loads, since more cores can be running concurrently within the chip's power envelope, the result is a higher throughput.

A roadblock for NTC is its higher sensitivity to process variations - i.e., the deviation of device parameters from their nominal values. Already in current-technology STC multicores, process variations result in noticeable differences in power and performance across the different cores of a chip [11]. At NTC, due to the low operating $V_{d d}$ [28], the same amount of process variations causes a substantially larger impact on transistor speed and power consumption variations. Process variations are undesirable because they result in chips that consume more static power, cycle at lower frequencies, and can even be faulty.

Process variations should be addressed at multiple levels, including at the computer architecture level. To confront variations at the architecture level, we first need models of process variations and how they affect frequency and power, at a level of abstraction that is useful to microarchitects. Such models exist for STC (e.g., [20], [25], [27], [35], [37]). Unfortunately, none of them is applicable to NTC - NTC uses new memory structures and requires new delay and power models.

This paper presents the first microarchitectural model of process variations for NTC. The model, called VARIUS-NTV, extends the existing VARIUS variation model [37]. It models how variation affects the frequency attained and power consumed by cores and memories in an NTC manycore, and the timing and stability faults in SRAM cells at NTC. The key aspects include: (i) adopting a gate-delay model and an SRAM cell type that are tailored to NTC, (ii) modeling SRAM failure modes emerging at NTC, and (iii) accounting for the impact of leakage current in SRAM timing and stability models.

We evaluate a simulated $11 \mathrm{~nm}, 288$-core tiled manycore at both NTC and STC. Our results show that the expected process variations induce higher differences in frequency $(f)$ and power at NTC than at STC. For example, the maximum difference in tile $f$ within a chip is $\approx 3.7 \mathrm{x}$ at NTC and only $\approx 2.3 \mathrm{x}$ at STC. We evaluate different core- 
tiling organizations in the chip and different configurations of on-chip $V_{d d^{-}}$and $f$-domains. Our experiments show that variation management is especially important at NTC. Finally, we validate our model against an experimental 80-core prototype chip [11].

This paper is organized as follows: Section II provides a background; Section III presents our VARIUS-NTV variation model; Section IV describes the manycore architecture evaluated; Sections V and VI evaluate VARIUS-NTV for the architecture; Section VII outlines our initial validation of VARIUS-NTV; and Section VIII discusses related work.

\section{BACKGROUND}

\section{A. Near-Threshold Computing (NTC) Basics}

NTC refers to an environment where $V_{d d}$ is set to a value only slightly higher than the transistors' $V_{t h}$ [7], [13], [28]. For current technologies, this roughly corresponds to $V_{d d} \approx 500 \mathrm{mV}$, while the $V_{d d}$ of conventional (or STC) environments is $\approx 1 \mathrm{~V}$.

NTC pushes back the manycore power wall by reducing the energy per operation several times compared to STC at the expense of degrading the frequency of operation [13]. The result is that the power is expected to reduce by about an order of magnitude, allowing more cores to operate simultaneously for the same manycore power envelope. If the application has parallelism, this is a major advantage.

Figure 1 compares the scaling of three parameters under NTC, STC, and as imposed by classical CMOS theory [10]: supply voltage, transistor delay and power density. The $\mathrm{X}$ axis shows gate length to characterize each technology generation. Classical scaling relies on scaling $V_{d d}$ down at every technology generation by a constant scaling factor $\kappa$. Both $V_{d d}$ and transistor delay reduce at each generation, giving rise to a constant power density. Conventional STC scaling deviates from classical scaling in that the decrease of the transistor's $V_{t h}$ has practically stopped to keep subthreshold leakage under control, which in turn has prevented $V_{d d}$ from scaling [19]. A consequence of this fact is that power density now keeps increasing. As we go from STC to NTC scaling, the curves experience vertical shifts. Specifically, as $V_{d d}$ decreases (Figure 1(a)), power density goes down and transistor delay increases (Figure 1(b)).

In terms of energy and delay, NTC is close to a sweet spot. Figure 2 shows the inverse of energy per operation (labeled as energy efficiency) in MIPS/Watt (left $\mathrm{Y}$ axis) and the transistor delay (right $\mathrm{Y}$ axis) as a function of $V_{d d}$. In the NTC region, the energy efficiency is high and the transistor delay is relatively low. Away from this region, higher $V_{d d}$ quickly results in substantially lower energy efficiency. Lower $V_{d d}$, on the other hand, quickly results in slower transistors.

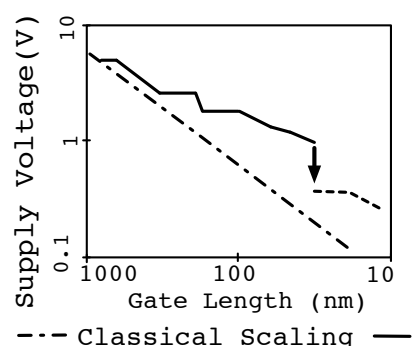

(a)

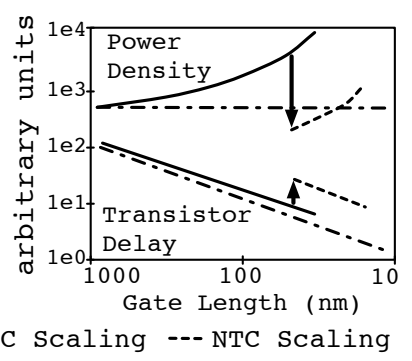

(b)
Figure 1. Parameter scaling under three scenarios [7].

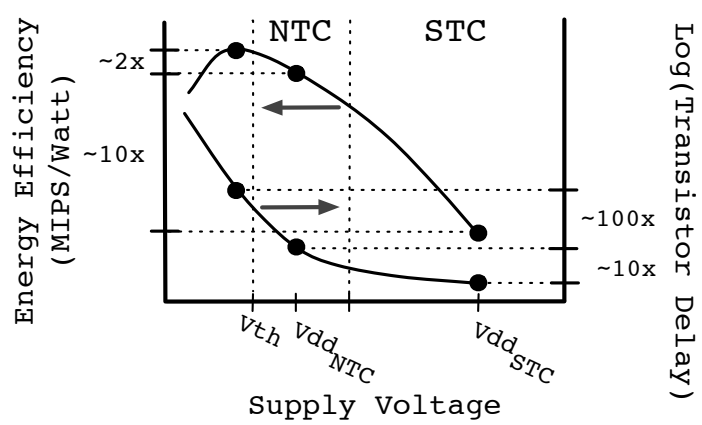

Figure 2. Impact of $V_{d d}$ on energy efficiency and delay [13].

\section{B. The Impact of Process Variations at NTC}

Each technology generation becomes increasingly vulnerable to process variations, which manifest across the chip as static, spatial fluctuations in transistor parameters around the nominal values [2], [3]. Within-die (WID) process variations are caused by systematic effects (e.g., due to lithographic irregularities) and random effects (e.g., due to varying dopant concentrations) [38]. Two key process parameters affected by variations are $V_{t h}$ and the effective channel length $\left(L_{e f f}\right)$. The higher the $V_{t h}$ and $L_{e f f}$ variations are, the higher the variations in transistor switching speed and static power consumption are. This results in chips with increased variation in frequency and power consumption across cores and memories. Note that, in an environment with variation, the average core has lower frequency than before. This is because the slower transistors determine the frequency of the whole core. Moreover, the average core consumes more static power. The reason is that low- $V_{t h}$ transistors consume more additional power than high- $V_{t h}$ ones save.

Unfortunately, transistor delay and power consumption are more sensitive to variations in $V_{t h}$ and $L_{e f f}$ at NTC than at STC. Consider transistor delay first. At low $V_{d d}$, transistor delay is experimentally found to be more sensitive to changes in $V_{t h}$ [14]. For example, Figure 3 shows the transistor delay from the model of Markovic et al. [28] as $V_{t h}$ varies. For $V_{d d}=0.6 \mathrm{~V}$, the difference in delay between 


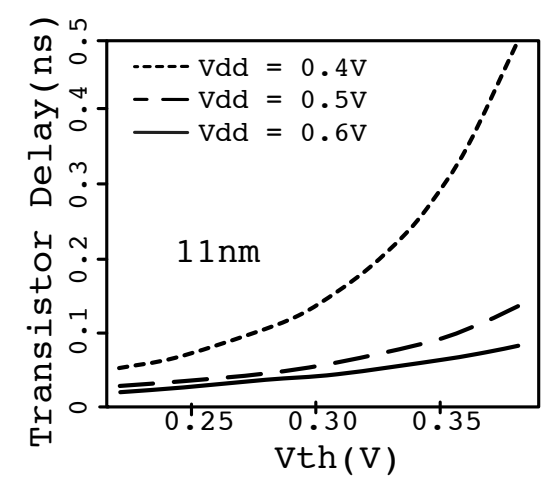

Figure 3. Transistor delay for different $V_{t h}$.

transistors of $V_{t h}=0.25 \mathrm{~V}$ and $0.35 \mathrm{~V}$ is around $30 \mathrm{ps}$, while for $V_{d d}=0.4 \mathrm{~V}$, it jumps to over $200 \mathrm{ps}$.

Dynamic power is also more sensitive to process variations at NTC than at STC. The reason is that dynamic power depends on the frequency and, as we have seen, at low $V_{d d}$, transistor delay (and hence frequency) is more sensitive to changes in $V_{t h}$.

\section{Modeling Process Variations at STC: VARIUS}

There are several microarchitectural models that analyze the impact of process variations on processors and memories at a level that is useful to microarchitects (e.g., [20], [25], [27], [35], [37]). However, these works only apply to STC, and not to NTC. In this paper, we take one of these models, namely VARIUS [37], and substantially extend it so that it applies to NTC. To understand our contributions, we briefly describe VARIUS.

VARIUS models variations in $V_{t h}$ and $L_{e f f}$. It models their systematic component by dividing the die into a grid and assigning to each grid point a $\Delta V_{t h}$ and $\Delta L_{e f f}$ value as sampled from a multivariate normal distribution with $\mu=0$ and $\sigma_{\text {sys }}$. Moreover, these values have a spatial correlation that follows a spherical function. With this function, the correlation between two points only depends on their Euclidean distance. At a distance equal to zero, the correlation is one. The correlation then decreases with distance and, at a distance called Correlation Range $(\phi)$, the correlation becomes zero. VARIUS models the random component of variation with a normal distribution with $\mu=0$ and $\sigma_{\text {ran }}$.

VARIUS plugs the $V_{t h}$ and $L_{e f f}$ variations in the alphapower law (Equation 1) and in the equation for static power [9]. It then finds the variation in transistor (and gate) delay and transistor static power, respectively.

$$
t_{g} \propto \frac{V_{d d} \times L_{e f f}}{\mu\left(V_{d d}-V_{t h}\right)^{\alpha}}
$$

To find the distribution of delay of a pipeline stage, VARIUS proceeds differently depending on whether the stage has only logic, only an SRAM memory access, or a combination of both. For logic, it assumes that wire delays do not suffer from variations and, knowing the number of gates in a logic path, it uses the gate delay variation computed above to compute the path delay variation. If VARIUS knows the distribution of the logic path delays in the stage (e.g., from Razor data [16]), it can estimate the distribution of variation-afflicted logic path delays.

For a stage with a memory access, VARIUS models the 6-transistor SRAM cell of Figure 4(a). Using the variation in transistor delay, it computes the variation in cell read access time. It assumes that the read access time is more critical than the write access time. Then, using the cell access time, it computes the memory line access time. Note that the pipeline stage also contains some logic, namely the decoder, the logic at the intersection of word- and bit-line, and the logic at the sense amplifier. The delay through all this logic is modeled using the previous logic-stage model and is added to the memory access delay to find the distribution of total path delay in the stage.

For pipeline stages that combine both logic and memory access, VARIUS estimates the delay distribution by appropriately weighting the delay of a logic stage and a memory stage. Finally, the pipeline stage with the longest delays determines the safe frequency of the processor.

The static power $\left(P_{s t a}\right)$ in the processor (or memory module) is found by integrating the $P_{\text {sta }}$ of all of its transistors. VARIUS uses statistical principles to find a normal distribution for the processor's $P_{s t a}$ as a function of the normal distributions of the transistors' $P_{s t a}$.

\section{VARIUS-NTV: A MiCROARCHITECTURAL MODEL OF PROCESS VARIATIONS FOR NTC}

VARIUS-NTV builds on VARIUS [37] to develop a microarchitectural model of process variations and resulting timing errors that is valid at NTC. Much of the general approach that VARIUS uses still applies to NTC - although the values of most parameters change. However, there are several important aspects that require complete redesign. This is where VARIUS-NTV contributes.

The main contributions of VARIUS-NTV are in four dimensions, which address four major limitations of VARIUS: (i) the VARIUS model for gate delay is based on the alphapower law, which is only accurate for $V_{d d}$ much larger than $V_{t h}$; (ii) the VARIUS memory model uses a 6-transistor SRAM cell, which cannot reliably operate at NTC; (iii) for SRAM cells, the VARIUS model only considers read access (or timing) failures, while other memory failure modes dominate at NTC; and (iv) in the SRAM failure analysis, VARIUS neglects the impact of leakage while, at NTC, the impact of leakage is substantial.

In this section, we present the main contributions of VARIUS-NTV. 


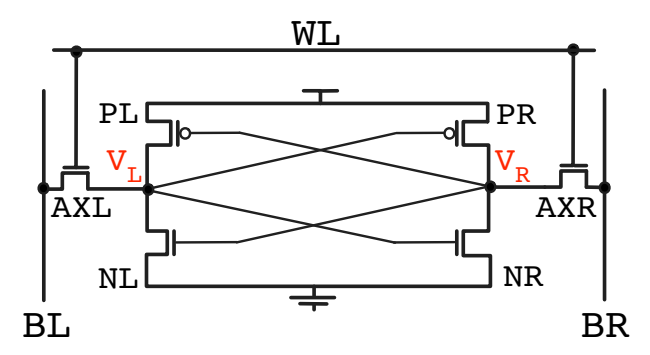

(a)

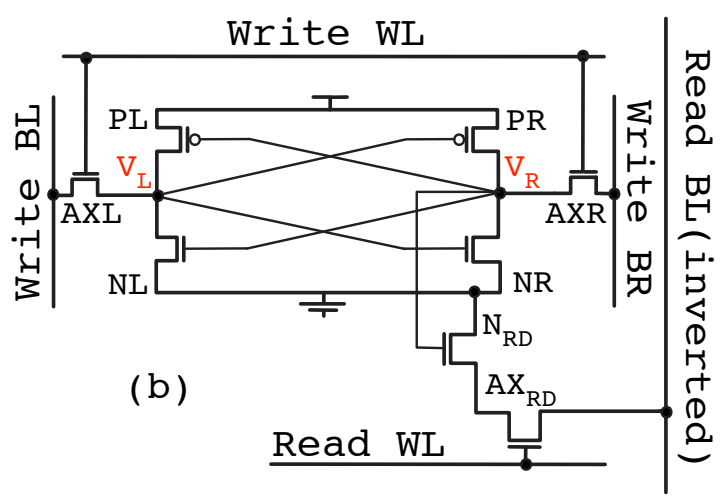

Figure 4. SRAM cell architecture: conventional 6-transistor cell (a) and 8-transistor cell (b). $V_{R}$ and $V_{L}$ are the voltages at the nodes indicated, which are referred to as nodes $R$ and $L$, respectively.

\section{A. Gate Delay}

To model the gate delay $\left(t_{g}\right)$, VARIUS uses the alphapower law (Equation 1), where $\alpha$ is a process parameter capturing carrier velocity saturation, and $\mu$ identifies the carrier mobility as a function of the temperature $(\mathrm{T})$. This equation does not model the NTC region accurately. There are alpha-power law variants [4], [6], [21], [31] that attempt to extend the model to the subthreshold region. Usually, they come with an increased number of fitting parameters that have no direct physical interpretation. Furthermore, that they cover the subthreshold region does not necessarily imply that they model NTC properly.

Consequently, in VARIUS-NTV, we use the EKVbased [15] model proposed by Markovic et al. [28]. The formula for the on-current is given in Equation 2, where $v_{t}$ is the thermal voltage and $n$ a process-dependent parameter determined by subthreshold characteristics. The carrier mobility's $T$ dependence is $\mu \propto T^{-1.5}$.

$$
I \propto \mu / L_{e f f} \times n \times v_{t}^{2} \times \ln ^{2}\left(e^{\frac{V g s-V t h}{2 \times n \times v_{t}}}+1\right)
$$

The resulting gate delay, obtained from $C V / I$, is shown in Equation 3. The equation captures the variation in gate delay as a function of the variation in $V_{t h}$ and $L_{e f f}$. Since the EKV model covers all regions of operation, Equation 3 is equally valid at STC and NTC. In all cases, $V_{t h}$ is a function of $V_{d d}$ and temperature as per Equation 4, where $V_{t h_{0}}, V_{d d_{0}}$ and $T_{0}$ are the nominal values of these parameters, and $k_{T}$ and $k_{D I B L}$ represent constants of proportionality capturing the impact of $T$ and DIBL (Drain Induced Barrier Lowering) on $V_{t h}$, respectively.

$$
\begin{array}{r}
t_{g} \propto \frac{V_{d d} \times L_{e f f}}{\mu \times n \times v_{t}^{2} \times \ln ^{2}\left(e^{\frac{V d d-V t h}{2 \times n \times v_{t}}}+1\right)} \\
V_{t h}=V_{t h_{0}}+k_{D I B L}\left(V_{d d}-V_{d d_{0}}\right)+k_{T}\left(T-T_{0}\right)
\end{array}
$$

\section{B. SRAM Cell}

VARIUS uses the conventional 6-transistor cell shown in Figure 4(a). This cell requires careful sizing of the transistors, since it poses conflicting requirements on the $A X R$ and $A X L$ access transistors to prevent both read and write failures. While such a design is typical at STC, it becomes very hard to use at NTC, where transistors are more sensitive to process variations. One way to address this problem is to power SRAMs at a higher $V_{d d}$ than the logic. Unfortunately, this approach is costly, since cache memory and logic blocks are often highly interleaved in the layout. Moreover, it requires extra voltage regulators in the platform, and results in additional design, validation, and testing issues. Finally, it is hardly scalable: as we move to smaller technologies, the relative difference between the safe SRAM and logic voltages increases, diminishing the power reduction benefit of NTC.

Consequently, VARIUS-NTV uses the 8-transistor cell of Figure 4(b) [8], [29]. This cell is easier to design reliably because it decouples the transistors used for reading $\left(A X_{R D}\right.$ and $N_{R D}$ ) and those for writing (the rest). Compared to the 6-transistor cell, read and write timing margins can be independently optimized with marginal increase in cell area [8]. In addition, of the five types of SRAM failure modes (read timing, read upset, write stability, write timing, and hold) [30], this cell eliminates read upset failures because the cell's internal nodes are decoupled from the read bit-line (BL).

\section{Memory Failure Modes}

While VARIUS only considers read timing failures, VARIUS-NTV models all of the SRAM failure modes (except read upsets, which cannot occur in the 8-transistor cell because a read cannot flip the cell contents by construction). We now describe how VARIUS-NTV models them.

1) Hold Failure: In a cell storing $0\left(V_{R}=0, V_{L}=1\right)$, at low $V_{d d}$, the voltage $V_{L}$ decreases by construction. This is 
because, when the cell is not accessed, although $N L, P R$, and the access transistors are off, there is leakage through $N L$ and $A X L$. A hold failure occurs when the leakage current through the $N L$ and $A X L$ transistors in Figure 4(b) reduces $V_{L}$ below the $V_{S W I T C H}$ of the $P R-N R$ inverter while the cell is not being accessed. At that point, the cell's state is lost.

To model these failures at a given $V_{d d}$, VARIUS-NTV uses Kirchhoff's current law to compute $V_{L}$ and $V_{S W I T C H}$ at $V_{d d} . V_{L}$ is extracted from $I_{P L}\left(V_{L}\right)-I_{N L}\left(V_{L}\right)-$ $I_{A X L}\left(V_{L}\right)=0$, where

$$
\begin{aligned}
I_{P L}\left(V_{L}\right) & \propto \mu / L_{e f f} \times n \times v_{t}^{2} \times \ln ^{2}\left(e^{\frac{V d d-V t h}{2 \times n \times v_{t}}}+1\right) \\
I_{N L}\left(V_{L}\right) & \propto \mu / L_{e f f} \times T^{2} \times e^{-\frac{V t h}{n \times v_{t}}} \\
I_{A X L}\left(V_{L}\right) & \propto \mu / L_{e f f} \times T^{2} \times e^{-\frac{V t h}{n \times v_{t}}}
\end{aligned}
$$

and where $V_{t h}$ in each equation is expressed as a (different) function of $V_{L}$.

Similarly, $V_{S W I T C H}$ is extracted from $I_{P R}\left(V_{S W I T C H}\right)-$ $I_{N R}\left(V_{S W I T C H}\right)+I_{A X R}\left(V_{S W I T C H}\right)=0$ for the $P R-N R$ inverter when $V_{I N}=V_{O U T}[30]$.

The hold failure probability of a cell is $P_{\text {Cell,Hold }}=$ $P\left[V_{L}\left(V_{d d}\right)-V_{S W I T C H}\left(V_{d d}\right)<0\right]$. Then, the hold failure probability of a line is $P_{\text {Line, Hold }}=1-(1-$ $\left.P_{C e l l, H o l d}\right)^{\text {line_size }}$, where line_size is the number of cells per line, and $1-\left(1-P_{C e l l, H o l d}\right)^{\text {line_size }}$ gives the probability that at least one cell fails. A line is faulty if at least one of its cells is faulty. The failure probability of cells is assumed independent in this case.

To avoid hold failures, the minimum allowable supply voltage, $V_{d d M I N, C e l l}$, is obtained by solving $V_{L}\left(V_{d d M I N, C e l l}\right)=V_{S W I T C H}\left(V_{d d M I N, C e l l}\right)$ under variation. Then, $V_{d d M I N, \text { Line }}=\max \left(V_{d d M I N, C e l l}\right)$ for all the cells in the line.

2) Write Stability Failure: Without loss of generality, we focus on a cell that stores a $0 \quad\left(V_{R}=0\right.$ and $\left.V_{L}=1\right)$. VARIUS-NTV computes the voltage $\left(V_{L W}\right)$ that node $L$ reaches when the write $B L$ is set to 0 (where $B R=1$ ) and the write duration is extended to infinity. If the value is above the switching threshold of the $P R-N R$ inverter $\left(V_{S W I T C H}\right)$, then a write failure occurs.

The $V_{L W}$ distribution is computed using Kirchhoff's current law at node $L$, from $I_{P L}\left(V_{L W}\right)-I_{N L}\left(V_{L W}\right)-$ $I_{A X L}\left(V_{L W}\right)=0$. The $V_{S W I T C H}$ distribution is extracted as explained above in the hold failure analysis.

In all cases, transistor parameters are subjected to the variation model. Finally, the per-cell probability of write stability failure becomes $P_{C e l l, W S t a b}=P\left[V_{L W}-V_{S W I T C H}>0\right]$. A memory line suffers from write stability failure if there is at least one cell in the line suffering from it.

3) Read Timing Failure: VARIUS-NTV computes the random variable that captures the time taken to generate a detectable voltage drop on the read bit-line as

$$
D_{\text {VarReadCell }} \propto \frac{1}{I_{A X_{R D}}+\sum I_{S T A}}
$$

where $I_{A X_{R D}}$ is the bit-line discharge current through the $A X_{R D}$ transistor in Figure 4(b), and $\sum I_{S T A}$ is the leakage over all of the cells attached to the bit-line. To calculate the distribution of $1 / I_{A X_{R D}}$, first, the source voltage of $A X_{R D}$, $V_{R D}$, is extracted by solving the Kirchhoff's law at this node, from $I_{A X_{R D}}\left(V_{R D}\right)=I_{N_{R D}}\left(V_{R D}\right)$. When reading from a cell storing $1\left(V_{R}=1\right.$ and $\left.V_{L}=0\right)$, the transistor currents follow from:

$$
\begin{aligned}
I_{A X_{R D}} & \propto \mu / L_{e f f} \times n \times v_{t}^{2} \times \ln ^{2}\left(e^{\frac{V d d-V_{R D}-V t h}{2 \times n \times v_{t}}}+1\right) \\
I_{N_{R D}} & \propto \mu / L_{e f f} \times n \times v_{t}^{2} \times \ln ^{2}\left(e^{\frac{V d d-V t h}{2 \times n \times v_{t}}}+1\right)
\end{aligned}
$$

where $V_{t h}$ in each equation is expressed as a (different) function of $V_{R D}$.

Then, the probability distribution of $D_{\text {VarReadCell }}$ can be attained by applying those of $V_{t h}$ and $L_{\text {eff }}$ given by

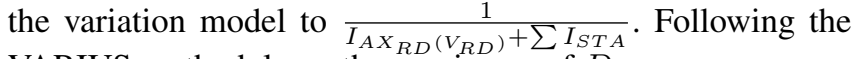
VARIUS methodology, the maximum of $D_{\text {VarReadCell }}$ over all of the cells in a line is the time to read an entire memory line $D_{V \text { arReadLine }}$. Finally, the probability of read access failure $\left(P_{\text {ReadAccess }}\right)$ is $P\left[D_{\text {VarReadLine }}>t_{R E A D}\right]$, where $t_{R E A D}$ is the designated read duration.

4) Write Timing Failure: Given a cell without write stability failure, VARIUS-NTV models a write timing failure by computing $D_{\text {VarWriteCell }}$. This is the time that node $L$ takes to reach the switching threshold $\left(V_{S W I T C H}\right)$ of the $P R-N R$ inverter. It is:

$$
\begin{gathered}
D_{\text {VarWriteCell }} \propto \frac{1}{I_{L}}=\int_{V d d}^{V_{S W I T C H}} d v_{L} / i_{L}\left(v_{L}\right) \\
i_{L}\left(v_{L}\right)=i_{P L}\left(v_{L}\right)-i_{N L}\left(v_{L}\right)-i_{A X L}\left(v_{L}\right)
\end{gathered}
$$

where $I_{L}$ is the discharge current at node $L$ during the write, obtained following [30]. $i_{L}\left(v_{L}\right)$ is a function of Gaussian random variables $V_{t h}$ and $L_{e f f}$ under process variation. It is obtained with Kirchhoff's current law.

After obtaining the probability distribution for $D_{\text {VarWriteCell, }}$ we compute the distribution of the maximum of $D_{\text {VarWriteCell }}$ over all of the cells in a line. Finally, the probability of write timing failure ( $\left.P_{W \text { riteTiming }}\right)$ is $P\left[D_{\text {VarWriteLine }}>t_{W R I T E}\right]$, where $t_{W R I T E}$ is the designated write duration.

\section{Impact of Leakage}

At NTC, the magnitude of the leakage current $\left(I_{o f f}\right)$, decreases when compared to STC. However, the on-current $\left(I_{o n}\right)$, decreases even more due to lower $V_{d d}$. Hence, the relative impact of $I_{o f f}$ increases. Consequently, unlike VARIUS, VARIUS-NTV takes into account the impact of the leakage current on SRAM timing and stability, as we have seen in previous sections. As part of $I_{o f f}$, we only consider 
subthreshold leakage; we exclude gate leakage because we assume high-K metal gate devices like the ones currently in use.

\section{MANycore ARChitecture Modeled}

To evaluate VARIUS-NTV, we model an $11 \mathrm{~nm}$ manycore architecture that operates at NTC. The manycore is organized in tiles (36 in our default configuration) for ease of design (Figure 5). Each tile has a tile memory and several cores (8 in our default configuration), each with a percore memory. Each core is a single-issue engine where memory accesses can be overlapped with each other and with computation. Each tile memory is a bank of a shared L2 cache, while the per-core memories are L1 caches. Data in the L1 caches is kept coherent with a directory-based MESI coherence protocol where each pointer corresponds to one tile. The cores are connected with a bus inside each tile and with a 2D torus across tiles. Table I shows the default architecture and technology parameters. In the table, all of the parameters that are not labeled with STC refer to the NTC environment.

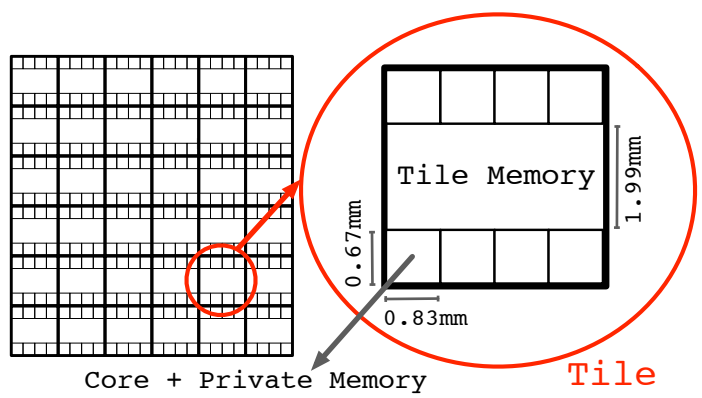

Figure 5. Manycore architecture used to evaluate VARIUS-NTV.

We evaluate an STC version of the manycore and three NTC versions of it. The three NTC versions differ based on the use of voltage and frequency domains, as listed in Table II.

The technology parameters used in Table I are derived from ITRS [22] and projected trends from industry. Every single experiment is repeated for 100 chips with different variation profiles, and we present the average. More samples beyond 100 do not change the results noticeably.

\section{EXPERIMENTAL SETUP}

We evaluate VARIUS-NTV by using it to estimate the performance and power consumption of the manycore architecture of Section IV. We interface Pin [26] over a user-level pthreads library to the SESC [34] cycle-level architectural simulator. SESC estimates both execution time and energy consumed. The energy analysis relies on McPAT [24] scaled to $11 \mathrm{~nm}$. An updated version of HotSpot takes the detailed

\begin{tabular}{|c|c|}
\hline \multicolumn{2}{|c|}{ System Parameters } \\
\hline $\begin{array}{l}\text { Technology node: } 11 \mathrm{~nm} \\
\text { Num. Cores: } 288 \\
\text { Num. Tiles: } 36 \text { ( } 8 \text { cores/tile })\end{array}$ & $\begin{array}{l}P_{M A X}=100 \mathrm{~W} \\
T_{M A X}=100^{\circ} \mathrm{C} \\
\text { Chip area } \approx 20 \mathrm{~mm} \times 20 \mathrm{~mm}\end{array}$ \\
\hline \multicolumn{2}{|c|}{$\begin{array}{ll}\text { Variation Parameters } \\
\end{array}$} \\
\hline $\begin{array}{l}\text { Correlation range: } \phi=0.1 \\
\text { Total }(\sigma / \mu)_{V t h}=20 \% \text {; equal } \\
\text { contrib. systematic \& random }\end{array}$ & $\begin{array}{l}\text { Sample size: } 100 \text { chips } \\
\text { Total }(\sigma / \mu)_{L e f f}=10 \% \text {; equal } \\
\text { contrib. systematic \& random }\end{array}$ \\
\hline \multicolumn{2}{|c|}{ Technology Parameters } \\
\hline $\begin{array}{l}V d d_{N O M} \text { at } \mathrm{STC}=0.77 \mathrm{~V} \\
V t h_{N O M} \text { at } \mathrm{STC}=0.30 \mathrm{~V} \\
f_{N O M} \text { at } \mathrm{STC}=3.3 \mathrm{GHz} \\
f_{\text {interconnect }} \text { at } \mathrm{STC}=2.5 \mathrm{GHz} \\
k_{T}=-1.5 \mathrm{~m} V / K ; n=1.5\end{array}$ & $\begin{array}{l}V d d_{N O M} \text { at } \mathrm{NTC}=0.54 \mathrm{~V} \\
V t h_{N O M} \text { at } \mathrm{NTC}=0.33 \mathrm{~V} \\
f_{N O M} \text { at } \mathrm{NTC}=1.0 \mathrm{GHz} \\
f_{\text {interconnect }} \text { at } \mathrm{NTC}=0.8 \mathrm{GHz} \\
k_{D I B L}=-150 \mathrm{mV} / \mathrm{V}\end{array}$ \\
\hline \multicolumn{2}{|c|}{ Architectural Parameters } \\
\hline $\begin{array}{l}\text { Per-core memory: 64KB WT, } \\
\text { 4-way, 2ns access, 64B line } \\
\text { On-chip network: bus inside tile } \\
\text { and 2D-torus across tiles } \\
\text { Crossing a } f \text { domain boundary: } 2 \mathrm{~ns}\end{array}$ & $\begin{array}{l}\text { Tile memory: } 2 \mathrm{MB} \mathrm{WB}, \\
\text { 16-way, } 10 \mathrm{~ns} \text { access, } 64 \mathrm{~B} \text { line } \\
\text { Directory-based MESI } \\
\text { Avg. memory round-trip access } \\
\text { time (before contention): } \approx 80 \mathrm{~ns}\end{array}$ \\
\hline
\end{tabular}

Table I

ARCHITECTURE AND TECHNOLOGY PARAMETERS.

\begin{tabular}{||l|l||}
\hline \hline Name & NTC Manycore Configuration \\
\hline \hline$M V M F$ & Multiple $V_{d d}$ and multiple $f$ domains (one per tile). \\
$S V M F$ & Single chip-wide $V_{d d}$ domain and one $f$ domain per tile. \\
$S V S F$ & Single chip-wide $V_{d d}$ and $f$ domains. \\
\hline \hline
\end{tabular}

Table II

CONFIGURATIONS FOR THE NTC MANYCORE.

layout of the chip and models the temperature, in turn affecting the leakage energy in a feedback loop. VARIUS-NTV is implemented in R [40].

In our experiments, we run multi-programmed workloads that contain some or all of the following 8 PARSEC applications: blackscholes, ferret, fluidanimate, raytrace, swaptions, canneal, dedup, and streamcluster. Each application can run with 4,8 or 16 threads. For each application, we measure the complete parallel section (called Region of Interest or ROI) running the simsmall input data set.

\section{Evaluation}

In our evaluation, we first describe how we set the operating voltages and frequencies of the manycore, then assess the impact of process variations in NTC and STC environments, and then explore some design parameters.

\section{A. Computing the Operating Point}

To determine the operating $V_{d d}$ and $f$ at NTC, our model starts with SRAM blocks. Our goal is to estimate $V_{d d M I N}$, the minimum sustainable $V_{d d}$. It is set by hold and write stability failure analyses.

Our model first finds the minimum $V_{d d}$ needed to avoid hold failures, namely $V_{d d, \text { hold }}$. The $V_{d d, \text { hold }}$ distribution is attained by solving $V_{L}\left(V_{d d, \text { hold }}\right)=V_{S W I T C H}\left(V_{d d, \text { hold }}\right)$, where the former is the voltage at node $L$ (Figure 4(b)), while 
the latter is the switching threshold of the $P R-N R$ inverter. The chosen $V_{d d, h o l d}$ value is obtained at the $3 \sigma$ of the distribution - after approximating to a normal distribution. Our model then proceeds with write stability failure analysis, to guarantee that the chosen $V_{d d \text {,hold }}$ also avoids write stability failures. At this step, a higher $V_{d d}$ may emerge, if the write stability failure rate at $V_{d d \text {,hold }}$ remains higher than the target tolerable error rate. The resulting $V_{d d}$ is $V_{d d M I N}$.

Once $V_{d d M I N}$ is picked, VARIUS-NTV considers timing issues in order to set the $f$. The selected $f$ is determined by the slowest component of the chip, based on our model's analysis of path delay distributions at $V_{d d M I N}$. For logic blocks, the analysis follows that of VARIUS [37]. For SRAMs, it can be shown that, for the parameters considered, write timing requires longer delays than read timing for the same $V_{d d}$. This is consistent with the work of Abella et al. [1]. Hence, write timing analysis determines the path delays in each SRAM block. To determine the maximum path delay, VARIUS-NTV approximates the path delay distributions to normal ones and picks the $3 \sigma$ cut-off point. This maximum delay determines the $f$ at $V_{d d M I N}$.

\section{B. Impact of Process Variations at NTC and STC}

To examine the impact of WID process variations on the $f$ and power consumption at NTC and STC, we consider three types of on-chip blocks separately: logic (the core pipelines), small memories (the per-core local memories) and large memories (the per-tile memories). We do this because they have different critical path distributions. In all cases, the $f$ for a block is determined by finding the distribution of the path delays in the block at $V_{d d N O M}$ and then picking, as the period for the block, the delay at the $3 \sigma$ of the distribution. The power of the block is the sum of the static and dynamic components.

We consider intra-tile variations first. In each tile, we compute the ratio of the frequencies of the fastest and slowest pipelines in the tile. We then take the average of the ratios across all tiles (Intra Pipe). We repeat the same process for local memories in the tile to calculate Intra Mem. Finally, for the power consumption, we take the power ratio of highest to lowest consuming pipelines, and highest to lowest consuming local memories, to compute Intra Pipe and Intra Mem, respectively.

For inter-tile variations, we measure the ratio of the frequencies of the fastest and slowest tile memories on chip (Inter Mem). We then consider the frequency that each tile can support (the lowest frequency of its pipelines, local memories and tile memory), and compute the ratio of the frequencies of the fastest and slowest tiles (Inter Pipe+Mem). Finally, we repeat the computations for power (Inter Mem and Inter Pipe+Mem). We report the mean of the experiments for 100 chips.

Figure 6 compares these ratios for NTC and STC. Figure 6(a) shows the $f$ ratios. We observe that the frequency ratio of the fastest to the slowest blocks is substantially higher at NTC than at STC - for the same process variation profile. For example, Inter Pipe+Mem at NTC is 3.7, while it is only 2.3 at STC (Figure 6(a)). This is because a low $V_{d d}$ amplifies the effect of process variations on delay.

Figure 6(b) shows the power ratios. The variation in total power also increases at NTC. However, the relative difference in power ratios between NTC and STC is generally smaller than the relative difference in frequency ratios. The reason is that power includes both dynamic and static power, and the ratios for static power are the same for NTC and STC. Consequently, the relative difference in power ratios is smaller. Still, the absolute difference is significant. Consequently, the chip is more heterogeneous at NTC.

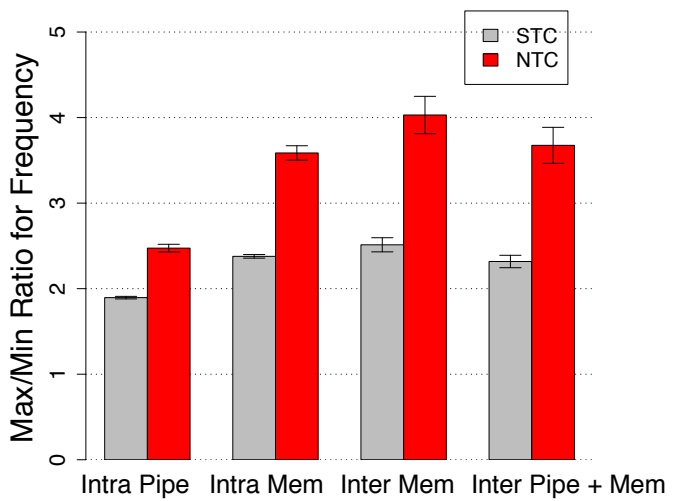

(a) Frequency

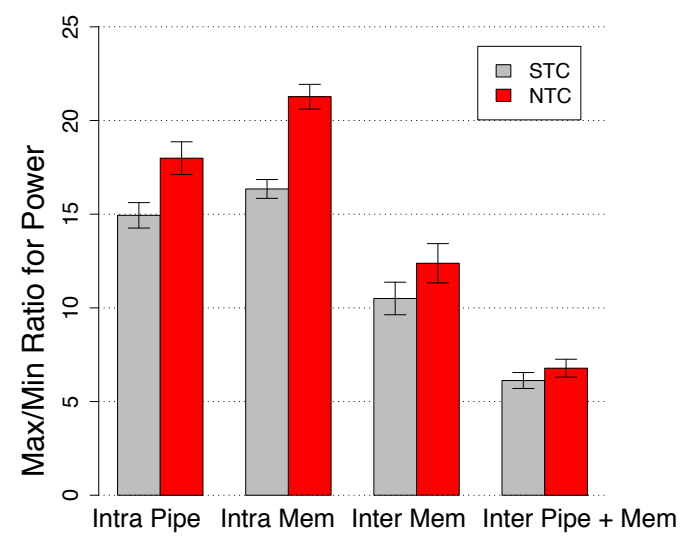

(b) Power

Figure 6. Impact of variations at NTC and STC.

These experiments have used a fixed, safe $V_{d d N O M}$ for the whole chip. In reality, process variations in the SRAM cells result in each tile supporting a different $V_{d d M I N}$, the minimum sustainable $V_{d d}$ to avoid failures. Such $V_{d d M I N}$ values are lower than $V_{d d N O M}$ for many tiles. Figure 7 shows the distribution of the $V_{d d M I N}$ values for all the tiles in a sample chip at NTC. The data is shown as a histogram. We can see that the $V_{d d M I N}$ values of tiles in a chip vary 
along a significant $0.46-0.58 \mathrm{~V}$ range.

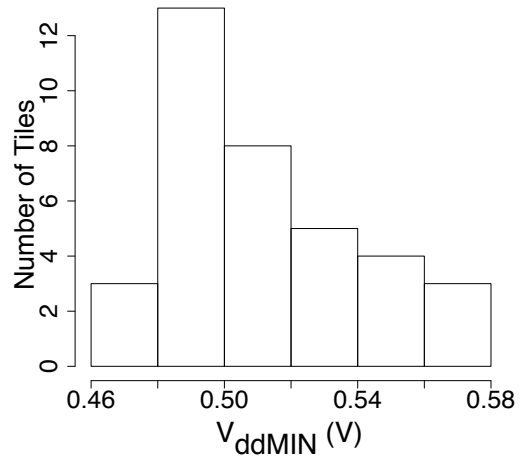

Figure 7. Values of $V_{d d M I N}$ for all the tiles of a representative chip at NTC.

\section{Design Space Exploration}

A promising way to combat the increased impact of process variations is to rely on fine grain, per-tile $V_{d d}$ and $f$ tuning. To quantify the effect, we compare the manycore configurations of Table II across different tile granularities ranging from 4 cores per tile to 16 cores per tile. $M V M F$ is an environment with a $V_{d d}$ and an $f$ domain per tile; $S V M F$ has a single $V_{d d}$ domain in the chip but one $f$ domain per tile; finally, SVSF characterizes a variation-oblivious environment, with a single $V_{d d}$ and $f$ domain per chip.

Figure 8 compares the performance (in normalized MIPS) of our 288-core NTC chip for the different environments. We consider two workload scenarios: one where we use all the tiles in the chip (Figure 8(a)) and one where we only use about half of the tiles (Figure $8(\mathrm{~b})$ ). Specifically, we use 128 out of the 288 cores and leave the others idle. Figure 9 repeats the analysis for STC.

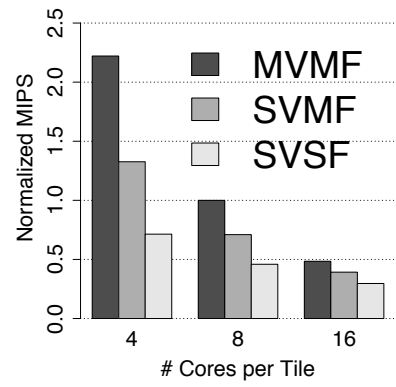

(a) $100 \%$ Use

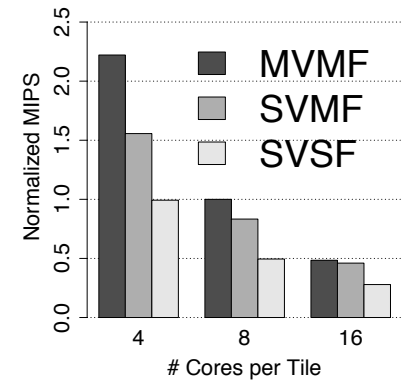

(b) $\approx 50 \%$ Use
Figure 8. Performance of our 288-core chip at NTC with different tile sizes and configurations. The charts correspond to using all the tiles (a) and using approximately only half (b).

In each figure, we keep the total number of cores in the chip constant, and perform a sensitivity analysis of different tile granularities: 4,8 or 16 cores per tile. In each case, the workload consists of 4-threaded, 8-threaded, or 16threaded parallel applications, respectively, from PARSEC. Each application uses one tile, and we report the average performance of the workload in MIPS. In each plot, to make the comparison fair, the power consumed by all of the environments is kept constant. In $M V M F$, the per-domain $V_{d d}$ and $f$ are set as per Section VI-A. Specifically, each tile runs at the tile-specific $V_{d d M I N}$, and at the maximum $f$ that it can support at this voltage. In $S V M F$, all the tiles in the chip run at the maximum of the $V_{d d M I N} \mathrm{~s}$ across all tiles. The per-tile frequencies are increased accordingly. Finally, in $S V S F$, the chip uses the same voltage as SFMV but it runs at the chip-wide minimum of per-tile frequencies. Recall that the $V_{d d M I N}$ of a tile represents the maximum $V_{d d M I N}$ across its components, where the $f$ of a tile corresponds to the minimum $f$ across its components at the designated tile $V_{d d}$. The applications are assigned to tiles according to highest average IPC application to highest $f$ tile. After the MIPS of each environment is computed, it is normalized to that of $M V M F$ for an 8-core tile in each plot.

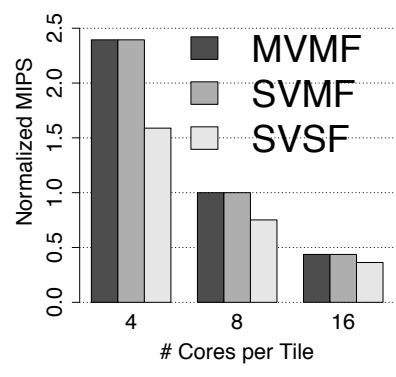

(a) $100 \%$ Use

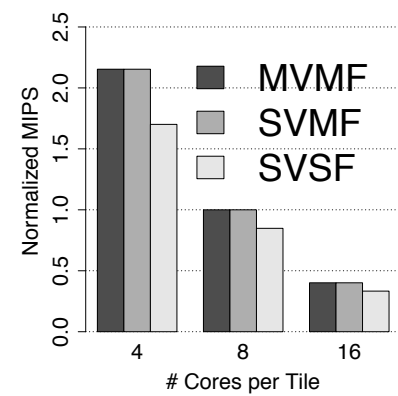

(b) $\approx 50 \%$ Use
Figure 9. Performance of our 288-core chip at STC with different tile sizes and configurations. The charts correspond to using all the tiles (a) and using approximately only half (b).

Starting with the fully-utilized chip (Figure 8(a)), we observe that SVMF only attains $59 \%, 71 \%$, and $81 \%$ MIPS of $M V M F$, for 4-core, 8-core, and 16-core tiles, respectively. This is because it does not exploit the multiple $V_{d d}$ domains of $M V M F$. The difference between the two bars gets larger as the tile granularity becomes finer, as $M V M F$ tracks core-tocore variations closer. SVSF in this case only reaches $32 \%$, $46 \%$, and $61 \%$ MIPS of $M V M F$, for 4-core, 8-core, and 16core tiles, respectively. As the tile granularity increases, the differences between the different configurations diminish.

Figure $8(\mathrm{~b})$ repeats the experiment when only $\approx$ half of the tiles are busy. For $M V M F$, we pick the 32, 16, and 8 most MIPS/W-efficient tiles for 4-, 8-, and 16-cores per tile granularity, respectively, and then assign the applications of higher IPC to the faster tiles in turn. The resulting power 
consumption is the power budget that we allow to the other environments. The other environments pick their 32,16 or 8 most MIPS/W-efficient tiles that satisfy the budget. We see similar trends as in Figure 8(a) except that the drop in MIPS is not as large. The reason is that each environment now picks a subset of energy-efficient tiles - leaving energyinefficient ones idle.

Finally, in Figure 9, the experiments are repeated for STC. For STC, $M V M F$ and $S V M F$ become equivalent, since the nominal STC $V_{d d}$ is high enough to produce a safe operating point across all of the tiles. There is no need to set the $V_{d d}$ of some tiles higher or lower depending on their $V_{d d M I N}$. Apart from this, while generally the same trends apply as under NTC operation, the MIPS loss as incurred by SVSF operation is much less.

\section{MOdel VALIDATION}

Our initial validation of VARIUS-NTV involves a validation of the parameters used and a comparison to the results reported in an experimental chip.

1) Validation of Model Parameters: VARIUS-NTV builds on the VARIUS variation and timing error model which, as explained in [37], was calibrated with experimental data from Friedberg et al. [17] and Razor [16], and validated with error rates in logic and memory [37]. To validate the new VARIUS-NTV formulas, we start with $V_{t h}$, which is a complex function of $V_{d d}$, Leff, and other technology parameters. We obtained a version of the $12 \mathrm{~nm}$ Predictive Technology Model (PTM) from Yu Cao from Arizona State University [32]. We compared the $V_{t h}$ values generated by VARIUS-NTV to those generated by the BSIM analytical model [5], and HSPICE. The $V_{t h}$ values from VARIUS-NTV closely track those from both HSPICE and BSIM with less than $1 \%$ error over the designated $V_{d d}$ range. The main source of discrepancy is the accuracy of modeling the DIBL effect.

We then used $V_{t h}$ values from VARIUS-NTV to generate values for gate delay and static power. We compared the values to HSPICE measurements of a FO4 inverter chain. The delay and static power scaling trends of VARIUS-NTV follow HSPICE within a $10 \%$ of error for our $V_{d d}$ range.

2) Comparison to Silicon Measurements: To further validate VARIUS-NTV, we compare its outputs to the variation measurements from Intel's 80-Core TeraFLOPS processor [11]. To this end, we experimented with a $12 \mathrm{~mm} \times 20 \mathrm{~mm}$ chip that mimicks the TeraFLOPS processor, where each core (which they call tile) has 2 floating point units, a $3 \mathrm{~KB}$ instruction memory, and a $2 \mathrm{~KB}$ data memory. According to the chip micrograph, the chip organizes the 80 cores into 10 rows and 8 columns. To match their technology parameters, we adapted VARIUS-NTV to a $65 \mathrm{~nm}$ CMOS technology with a $V_{d d N O M}$ of $1.2 \mathrm{~V}$.

Figure 8 in [11] depicts the measured variation in core frequency $\left(f_{M A X}\right)$ for the 80 cores of a single die at $50^{\circ} \mathrm{C}$ and $V_{d d}=0.8 \mathrm{~V}$. At $0.8 \mathrm{~V}$, the authors report a ratio of highest core frequency to lowest core frequency equal to 1.62 .

We repeat the conditions in which these measurements were taken to the extent that we can. We generate VARIUS-NTV frequency maps for 100 sample dies, assuming $(\sigma / \mu)_{V t h}=5 \%$ for the $65 \mathrm{~nm}$ technology, with an equal contribution of random and systematic variation. The histogram of the resulting ratios of highest core frequency to lowest core frequency as generated by VARIUS-NTV is shown in Figure 10(a). As shown in the histogram, VARIUS-NTV produces an average value of $\approx 1.48$ for the ratio of frequencies, with a $95 \%$ confidence interval of (1.452, 1.483).

Further, Figure 10(b) shows the frequency distribution of the cores in one of the dies, as generated by VARIUS-NTV at $0.8 \mathrm{~V}$. For this particular die, the ratio of highest core frequency to lowest core frequency is $\approx 1.4$. This figure is very similar to Figure 8 in [11].

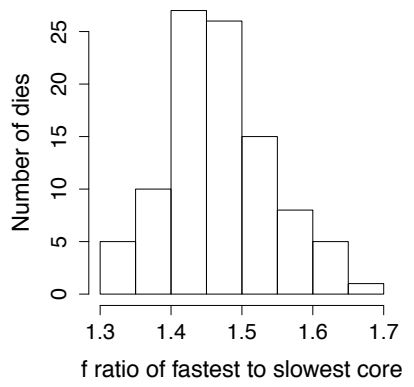

(a)

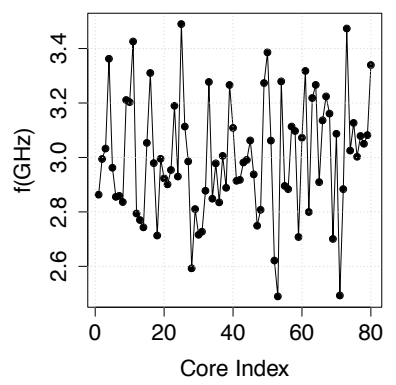

(b)
Figure 10. Data generated by VARIUS-NTV that replicates the data presented in [11]. Chart (a) shows a histogram of the ratios of highest core frequency to lowest core frequency over 100 dies. Chart (b) shows the frequency map for one of the sample dies.

Recall that the 80-Core processor does not represent an NTC design. However, our validation experiments are run at the relatively low $0.8 \mathrm{~V}$ (where the nominal $V_{d d}$ at $65 \mathrm{~nm}$ is $1.2 \mathrm{~V})$. No further measured data is provided in [11] below $0.8 \mathrm{~V}$. To our knowledge, there is no detailed variation characterization of any NTC chip that is available.

\section{RELATED WORK}

There are several microarchitectural models that analyze the impact of process variations on the frequency and power of processors and memories at a level that is useful to microarchitects. They include the work of Humenay et al. [20], Liang and Brooks [25], Marculescu and Talpes [27], Romanescu et al. [35], and Sarangi et al. [37] (on which this work builds) among others. As indicated before, these works only apply to STC, and not to NTC.

A few papers include a good description of the challenges and issues at NTC [7], [13], [28]. 
There are many other works that are related to evaluating the impact of process variation, mostly in STC environments. We list some of the most relevant here. Humenay et al. demonstrate that WID process variations lead to considerable performance and power consumption asymmetry among the cores in a CMP [20]. To minimize such asymmetry, they propose per-core ABB and ASV. Donald and Martonosi analyze core-to-core power variations in a CMP due to WID variation [12]. They propose to turn off cores when they consume excessive leakage power in order to maximize the chip-wide performance/power. Herbert and Marculescu examine the impact of core size on the throughput of a fixed area chip in the presence of WID variations [18]. They find that smaller cores (thus more cores per chip) running at independent $f$ lead to higher throughput than larger ones. Li and Martinez propose to optimize the number of active cores and their $V d d$ s and $f$ s jointly while running a workload on a CMP [23] where they apply DVFS chipwide rather than independently per core. In [33], Rangan et al. propose a throughput driven scheduling scheme to guarantee that a variation-afflicted chip performs very close to a perfect chip operating at the average frequency of the former. Rotem et al. [36] analyze the impact of single and multiple voltage and frequency domains in a CMP environment, considering power delivery limitations. They propose a clustered topology to maximize performance. The authors ignore the impact of variation. Finally, Teodorescu and Torrellas [39] examine the impact of process scheduling in the context of a manycore with variation. They provide heuristics to schedule the workload for performance or for power efficiency. It would be interesting to reproduce these works in the context of NTC.

\section{CONCLUSION}

To help confront process variations at the architecture level at NTC, this paper has presented the first microarchitectural model of process variations for NTC. The model, called VARIUS-NTV, extends an existing variation model for STC. It models how variation affects the frequency attained and power consumed by cores and memories in an NTC manycore, and the timing and stability faults in SRAM cells at NTC. The key aspects include: (i) adopting a gate-delay model and an SRAM cell type that are tailored to NTC, (ii) modeling SRAM failure modes emerging at NTC, and (iii) accounting for the impact of leakage in SRAM failure models.

We evaluated a simulated $11 \mathrm{~nm}$ manycore at both NTC and STC. Our results showed that the expected process variations induce higher differences in $f$ and power at NTC than at STC. For example, the maximum difference in tile $f$ within a chip is $\approx 3.7 x$ at NTC and only $\approx 2.3 x$ at STC. We evaluated different core-tiling organizations in the chip and different configurations of on-chip $V_{d d^{-}}$and $f$-domains. Our experiments showed that variation management is more crucial at NTC. Finally, we validated our model against an experimental 80-core prototype chip. We are ready to release the model and all its software to the public domain.

\section{REFERENCES}

[1] J. Abella, P. Chaparro, X. Vera, J. Carretero, and A. Gonzalez. High-Performance Low-Vcc In-Order Core. In International Symposium on High Performance Computer Architecture, January 2010.

[2] K. Bernstein, D. J. Frank, A. E. Gattiker, W. Haensch, B. L. Ji, S. R. Nassif, E. J. Nowak, D. J. Pearson, and N. J. Rohrer. High-Performance CMOS Variability in the 65-nm Regime and Beyond. In IBM Journal of Research and Development, July/September 2006.

[3] S. Borkar, T. Karnik, S. Narendra, J. Tschanz, A. Keshavarzi, and V. De. Parameter Variations and Impact on Circuits and Microarchitecture. In Design Automation Conference, June 2003.

[4] K. A. Bowman, B. L. Austin, J. C. Eble, X. Tang, and J. D. Meindl. A Physical Alpha-power Law MOSFET model. In International Symposium on Low Power Electronics and Design, August 1999.

[5] BSIM. http://www-device.eecs.berkeley.edu/ bsim/BSIM4.

[6] Y. Cao and L. T. Clark. Mapping Statistical Process Variations Toward Circuit Performance Variability: An Analytical Modeling Approach. In Design Automation Conference, June 2005.

[7] L. Chang, D. J. Frank, R. K. Montoye, S. J. Koester, B. L. Ji, P. W. Coteus, R. H. Dennard, and W. Haensch. Practical Strategies for Power-Efficient Computing Technologies. Proceedings of the IEEE, February 2010.

[8] L. Chang, R. Montoye, Y. Nakamura, K. Batson, R. Eickemeyer, R. Dennard, W. Haensch, and D. Jamsek. An 8TSRAM for Variability Tolerance and Low-Voltage Operation in High-Performance Caches. Journal of Solid-State Circuits, April 2008.

[9] Y. Cheng and C. Hu. MOSFET Modeling and Bsim3 User's Guide. Kluwer Academic Publishers, 1999.

[10] R. Dennard, F. Gaensslen, V. Rideout, E. Bassous, and A. LeBlanc. Design of Ion-Implanted MOSFETs with Very Small Physical Dimensions. In Journal of Solid-State Circuits, October 1974.

[11] S. Dighe, S. Vangal, P. Aseron, S. Kumar, T. Jacob, K. Bowman, J. Howard, J. Tschanz, V. Erraguntla, N. Borkar, V. De, and S. Borkar. Within-Die Variation-Aware Dynamic-VoltageFrequency-Scaling With Optimal Core Allocation and Thread Hopping for the 80-Core TeraFLOPS Processor. Journal of Solid-State Circuits, January 2011.

[12] J. Donald and M. Martonosi. Power Efficiency for Variationtolerant Multicore Processors. In International Symposium on Low power Electronics and Design, October 2006. 
[13] R. G. Dreslinski, M. Wieckowski, D. Blaauw, D. Sylvester, and T. Mudge. Near-Threshold Computing: Reclaiming Moore's Law Through Energy Efficient Integrated Circuits. Proceedings of the IEEE, February 2010.

[14] M. Eisele, J. Berthold, D. Schmitt-Landsiedel, and R. Mahnkopf. The Impact of Intra-die Device Parameter Variations on Path Delays and on the Design for Yield of Low Voltage Digital Circuits. Transactions on VLSI Systems, December 1997.

[15] C. C. Enz, F. Krummenacher, and E. A. Vittoz. An Analytical MOS Transistor Model Valid in All Regions of Operation and Dedicated to Low-voltage and Low-current Applications. Analog Integrated Circuits Signal Processing, 1995.

[16] D. Ernst, N. S. Kim, S. Das, S. Pant, R. Rao, T. Pham, C. Zeisler, D. Blaauw, T. Austin, K. Flautner, and T. Mudge. Razor: A Low-Power Pipeline Based on Circuit-Level Timing Speculation. In International Symposium on Microarchitecture, December 2003.

[17] P. Friedberg, Y. Cao, J. Cain, R. Wang, J. Rabaey, and C. Spanos. Modeling Within-die Spatial Correlation Effects for Process-design Co-optimization. In International Symposium on Quality of Electronic Design, March 2005.

[18] S. Herbert and D. Marculescu. Characterizing Chipmultiprocessor Variability-tolerance. In Design Automation Conference, June 2008.

[19] M. Horowitz, E. Alon, D. Patil, S. Naffziger, R. Kumar, and K. Bernstein. Scaling, Power, and the Future of CMOS. In International Electron Devices Meeting, December 2005.

[20] E. Humenay, D. Tarjan, and K. Skadron. Impact of Process Variations on Multicore Performance Symmetry. In Conference on Design, Automation and Test in Europe, April 2007.

[21] H. Im. Physical insight into fractional power dependence of saturation current on gate voltage in advanced short channel MOSFETS (alpha-power law model). In International Symposium on Low Power Electronics and Design, August 2002.

[22] International Technology Roadmap for Semiconductors (ITRS). 2009 Update.

[23] J. Li and J. Martinez. Dynamic power-performance adaptation of parallel computation on chip multiprocessors. In International Symposium on High-Performance Computer Architecture, February 2006.

[24] S. Li, J. H. Ahn, R. D. Strong, J. B. Brockman, D. M. Tullsen, and N. P. Jouppi. McPAT: An integrated power, area, and timing modeling framework for multicore and manycore architectures. In International Symposium on Microarchitecture, December 2009.

[25] X. Liang and D. Brooks. Mitigating the impact of process variations on processor register files and execution units. In International Symposium on Microarchitecture, December 2006.
[26] C.-K. Luk, R. Cohn, R. Muth, H. Patil, A. Klauser, G. Lowney, S. Wallace, V. J. Reddi, and K. Hazelwood. Pin: Building customized program analysis tools with dynamic instrumentation. In Conference on Programming Language Design and Implementation, June 2005.

[27] D. Marculescu and E. Talpes. Variability and energy awareness: A microarchitecture-level perspective. In Design Automation Conference, June 2005.

[28] D. Markovic, C. C. Wang, L. P. Alarcon, T.-T. Liu, and J. M. Rabaey. Ultralow-power design in near-threshold region. Proceedings of the IEEE, February 2010.

[29] Y. Morita, H. Fujiwara, H. Noguchi, Y. Iguchi, K. Nii, H. Kawaguchi, and M. Yoshimoto. An Area-Conscious LowVoltage-Oriented 8T-SRAM Design under DVS Environment. In Symposium on VLSI Circuits, June 2007.

[30] S. Mukhopadhyay, H. Mahmoodi, and K. Roy. Modeling of failure probability and statistical design of SRAM array for yield enhancement in nanoscaled CMOS. Transactions on Computer-Aided Design of Integrated Circuits and Systems, December 2005.

[31] M. Orshansky, J. Chen, and C. Hu. Direct sampling methodology for statistical analysis of scaled CMOS technologies. Transactions on Semiconductor Manufacturing, November 1999.

[32] Predictive Technology Model (PTM). http://ptm.asu.edu/.

[33] K. Rangan, M. Powell, G.-Y. Wei, and D. Brooks. Achieving uniform performance and maximizing throughput in the presence of heterogeneity. In International Symposium on High Performance Computer Architecture, February 2011.

[34] J. Renau, B. Fraguela, J. Tuck, W. Liu, M. Prvulovic, L. Ceze, S. Sarangi, P. Sack, K. Strauss, and P. Montesinos. SESC simulator, January 2005. http://sesc.sourceforge.net.

[35] B. F. Romanescu, S. Ozev, and D. J. Sorin. Quantifying the impact of process variability on microprocessor behavior. In Workshop on Architectural Reliability, December 2006.

[36] E. Rotem, R. Ginosar, A. Mendelson, and U. Weiser. Multiple clock and voltage domains for chip multi processors. In International Symposium on Microarchitecture, December 2009.

[37] S. Sarangi, B. Greskamp, R. Teodorescu, J. Nakano, A. Tiwari, and J. Torrellas. VARIUS: A model of process variation and resulting timing errors for microarchitects. Transactions on Semiconductor Manufacturing, February 2008.

[38] A. Srivastava, D. Sylvester, and D. Blaauw. Statistical Analysis and Optimization for VLSI: Timing and Power. Springer, 2005.

[39] R. Teodorescu and J. Torrellas. Variation-Aware Application Scheduling and Power Management for Chip Multiprocessors. In International Symposium on Computer Architecture, June 2008 .

[40] The R Project for Statistical Computing. http://www.rproject.org/. 